\title{
História de vida de Laetacara aff. araguaiae Ottoni \& Costa, 2009 (Perciformes, Cichlidae) em dois riachos no Noroeste do Estado de São Paulo, Brasil
}

\author{
Paulo Sérgio de Souza Filho ${ }^{1,2}$ \& Lilian Casatti ${ }^{1}$ \\ ${ }^{1}$ Departamento de Zoologia e Botânica, Instituto de Biociências, Letras e Ciências Exatas - IBILCE, \\ Universidade Estadual Paulista - UNESP, Rua Cristóvão Colombo, 2265, \\ CEP 15054-000, São José do Rio Preto, SP, Brasil \\ ${ }^{2}$ Autor para correspondência: Paulo Sérgio de Souza Filho,e-mail: paulounesp@gmail.com
}

SOUZA FILHO, P.S. \& CASATTI, L. Life history of Laetacara aff. araguaiae Ottoni \& Costa, 2009 (Perciformes, Cichlidae) in two streams in Northwestern São Paulo State, Brazil. Biota Neotrop. 10(2): http://www.biotaneotropica.org.br/v10n2/en/abstract?article+bn03910022010.

\begin{abstract}
The structure in size, density, feeding, fecundity, and presence of ectoparasites in two populations of Laetacara aff. araguaiae was investigated in two streams, in Northwestern São Paulo State, one structurally more heterogeneous (HET) and other relatively more simplified (SIM). Six samples were taken in each stream, three in the wet period (October, December/2007, February/2008) and three in the dry period (April, July, September/2008), which were performed during 15 minutes of sampling in each stream, using a $3 \mathrm{~mm}$-mesh sieve. A total of 118 individuals in HET stream (49 in the wet season and 69 in dry) and 146 in SIM stream were captured (82 in wet and 64 in dry). Fish collected in HET stream were larger than those collected in the SIM, both in wet and dry periods. The density was similar between two streams, with an increase agreeing with the peak of the dry period. Fragments of aquatic insects were the most important items of diet, which showed little spatial and temporal variation. Fecundity was significantly higher in HET, ranging from 977 to 1,602 oocytes, in contrast with females of the SIM, which had 338 to 814 oocytes. The ectoparasites (Digenea) were present only in specimens of the SIM, and were attached to the fins (dorsal and anal) with higher prevalence in October. Feeding and density were similar in the two populations of Laetacara aff. araguaiae, contrasting with the structure in size and fecundity. The differences in these demographic parameters do not occur randomly and are possibly reflecting the high rates of parasitism in the more simplified stretch that determines lower resources allocation to growth and reproduction of fishes in this population.
\end{abstract}

Keywords: structure in size, density, feeding, fecundity, ectoparasites, heterogeneity, upper Paraná.

SOUZA FILHO, P.S. \& CASATTI, L. História de vida de Laetacara aff. araguaiae Ottoni \& Costa, 2009 (Perciformes, Cichlidae) em dois riachos no Noroeste do Estado de São Paulo, Brasil. Biota Neotrop. 10(2): http://www.biotaneotropica.org.br/v10n2/pt/abstract?article+bn03910022010.

Resumo: Foi investigada a estrutura em tamanho, densidade, alimentação, fecundidade e presença de ectoparasitos em duas populações de Laetacara aff. araguaiae no Noroeste do Estado de São Paulo, em dois riachos, sendo um estruturalmente mais heterogêneo (HET) e outro relativamente mais simplificado (SIM). Foram realizadas seis amostragens em cada riacho, sendo três no período chuvoso (outubro, dezembro/2007, fevereiro/2008) e três no seco (abril, julho e setembro/2008), durante 15 minutos de coletas em cada riacho, utilizando-se uma peneira com malha de $3 \mathrm{~mm}$. Foram coletados 118 indivíduos no riacho HET (49 no período chuvoso e 69 no seco) e 146 no riacho SIM (82 no chuvoso e 64 no seco). Os peixes coletados no riacho HET foram maiores do que aqueles coletados no riacho SIM, independente do período considerado. A densidade populacional foi similar entre os dois riachos, com aumento que coincidiu com o pico do período seco. Fragmentos de insetos aquáticos representaram os itens mais importantes da dieta, com variação espaço-temporal pouco relevante. A fecundidade foi significativamente maior no riacho HET, variando de 977 a 1.602 ovócitos, em contraste com as fêmeas do riacho SIM, que apresentaram de 338 a 814 ovócitos. Os ectoparasitos (Digenea) estiveram presentes somente em indivíduos do riacho SIM e encontravam-se aderidos às nadadeiras (dorsal e anal), com maior prevalência no mês de outubro. A alimentação e densidade foram similares nas duas populações de Laetacara aff. araguaiae, ao contrário da estrutura em tamanho e fecundidade. As diferenças nestes parâmetros demográficos não ocorrem ao acaso e são possivelmente reflexo das elevadas taxas de parasitismo no riacho mais simplificado que determinam menor alocação de recursos para crescimento e reprodução nesta população de peixes.

Palavras-chave: estrutura em tamanho, densidade, alimentação, fecundidade, ectoparasitos, heterogeneidade, alto Paraná. 


\section{Introdução}

A heterogeneidade espacial em sistemas ecológicos é vista como um dos principais fatores que exercem influência sobre a dinâmica de populações (Hastings 1991), competição entre diferentes espécies (Bengtsson 1991), interações entre presas e predadores (Taylor 1990), disponibilidade de manchas de recursos (McIntosh et al. 2004) e organização de sistemas parasito-hospedeiro (Grosholz 1993). Em ambientes aquáticos, ela pode ser representada por combinações de rochas, troncos, galhos, macrófitas, algas e folhiço, além das variações locais de fluxo e profundidade da água (Matthews 1998). Existem várias abordagens que podem ser usadas para acessar a amplitude de processos e padrões que podem ser influenciados pelas muitas formas de heterogeneidade existentes em múltiplas escalas (Palmer \& Poff 1997).

Rios e riachos oferecem boas oportunidades para explorar a influência deste fator sobre diversos aspectos ecológicos. Dentre os estudos já realizados sob este enfoque, Theel et al. (2008) verificaram que a composição de comunidades de macroinvertebrados varia com a heterogeneidade espacial enquanto Simpson et al. (2008) registraram maior rapidez nas taxas de colonização por diatomáceas em riachos experimentais estruturalmente mais complexos. Dessa forma, estes estudos exemplificam que a influência da heterogeneidade ambiental se estende a diversos grupos biológicos e pode ser detectada em condições naturais ou experimentais.

Diversos estudos apontam forte associação de peixes em riachos com características locais que promovem complexidade estrutural em diferentes escalas (e.g., Gorman \& Karr 1978, Angermeier \& Karr 1984, Flecker 1997, Bührnheim \& Cox-Fernandes 2003), sendo que a simplificação de hábitats pode afetar diretamente diversos atributos ecológicos, desde populações (e.g., Torgersen \& Close 2004) a comunidades (e.g., Snyder et al. 2006, Schneider \& Winemiller 2008).

No sistema do alto rio Paraná, observações preliminares registraram elevada abundância de Laetacara aff. araguaiae (Figura 1) em áreas marginais rasas de riachos com condições estruturais diversas e até mesmo de hipoxia (Casatti et al. 2006). Apesar da espécie aparentemente demonstrar-se generalista quanto à ocupação de riachos, acredita-se que, em locais estruturalmente mais heterogêneos, alguns parâmetros populacionais e reprodutivos da espécie serão diferentes quando comparados o outros estruturalmente mais simplificadas, com menor disponibilidade de componentes estruturais que confiram sítios para abrigo e forrageio. Assim, no presente estudo investigou-se a estrutura em tamanho, densidade, alimentação, fecundidade e presença de ectoparasitos em duas populações de Laetacara aff. araguaiae em dois riachos de uma mesma bacia de drenagem no Noroeste do Estado de São Paulo, caracterizados por diferentes condições de heterogeneidade física do hábitat.

\section{Material e Métodos}

Os dois riachos estudados situam-se na porção noroeste do Planalto Ocidental Paulista, bacia do alto rio Paraná, em uma área de relevo suave, ondulado e razoavelmente uniforme (Arid 1973). O clima é tropical quente (Nimer 1989), com duas estações pluviométricas bem definidas: uma chuvosa, de outubro a março, e outra seca, de abril a setembro. Foi selecionado um trecho $\left(20^{\circ} 13\right.$ ' $12,7^{\prime \prime} \mathrm{S}$ e $50^{\circ} 30^{\prime} 11,0$ ” W) apresentando alta heterogeneidade de hábitat (HET) no córrego do Cedro no município de Vitória Brasil, e um trecho $\left(20^{\circ} 09^{\prime} 28,2\right.$ " $\mathrm{S}$ e $50^{\circ} 32$ ' 11,4 " $\mathrm{W}$ ) relativamente mais simplificado (SIM) de um riacho sem nome no município de Dolcinópolis (Figura 2). Ambos os riachos são trechos de primeira ordem, afluentes do ribeirão do Arara, que é um dos tributários do rio Grande, na divisa entre os Estados de São Paulo e Minas Gerais. O hábitat interno do riacho HET apresenta-se ocupado por Eichhornia crassipes (Mart.) Solms e Heteranthera reniformis Ruiz \& Pav., enquanto que o riacho SIM apresenta esparsas touceiras de Poaceae, em meio ao fundo de areia.

Foram realizadas seis amostragens em cada riacho, sendo três no período chuvoso (outubro, dezembro/2007, fevereiro/2008) e três no seco (abril, julho e setembro/2008). A cada amostragem foram caracterizados os descritores limnológicos (condutividade elétrica, temperatura, turbidez e largura, comprimento e profundidade em vários pontos, para posterior cálculo do volume de hábitat), com o objetivo de verificar possíveis alterações sazonais e espaciais. $\mathrm{O}$ esforço de amostragem foi padronizado em 15 minutos de coletas em cada riacho, com a utilização de uma peneira de malha fina ( $3 \mathrm{~mm}$ entre nós). Após a captura, os indivíduos foram fixados em formalina a $10 \%$ por um período de 48 horas e depois conservados em uma solução de EtOH a 70\%. Indivíduos testemunho estão depositados na Coleção de Peixes do Departamento de Zoologia e Botânica (DZSJRP) do Instituto de Biociências, Letras e Ciências Exatas de São José do Rio Preto, SP.

Em laboratório, os indivíduos foram pesados e medidos, sendo determinadas seis classes de tamanho (6,0-15,9; 16,0-25,9; 26,0-35,9; $36,0-45,9 ; 46,0-55,9 ; 56,0-65,9 \mathrm{~mm} \mathrm{CP}$ ) para os cálculos da estrutura em tamanho e densidade (por volume de hábitat) de cada população. A dieta foi determinada pela análise dos conteúdos gástricos. Os estômagos foram removidos e os itens alimentares triados e identificados, sendo que para cada item foi calculada a freqüência de ocorrência (Gelwick \& Matthews 1996) e a dominância (Hynes 1950), esta dada como a porcentagem do número de vezes em que o item ocupa o maior volume de cada estômago em função do número total de indivíduos analisados. Foi aplicada uma modificação do método gráfico de Costello (1990), na qual o volume/peso foi substituído pela dominância, a qual foi plotada no eixo y em função da freqüência de ocorrência no eixo x (Bennemann et al. 2006). A partir desses gráficos foram determinados os itens alimentares mais importantes na dieta de cada espécie (os mais próximos à extremidade do quadrante superior direito) e, assim, definido o grupo trófico de cada população em cada período sazonal. O grau de enchimento dos estômagos foi determinado por estimativa visual, sendo definido a partir de três estados possíveis: (1) estômago vazio, (2) parcialmente cheio e (3) cheio. A fecundidade foi determinada com a contagem de ovócitos em 16 fêmeas maduras. Todos os indivíduos foram examinados para avaliar a presença de ectoparasitos que, quando presentes, foram extraídos, contados e com essas informações foram calculadas a abundância média, intensidade média e prevalência (Bush et al. 1997).

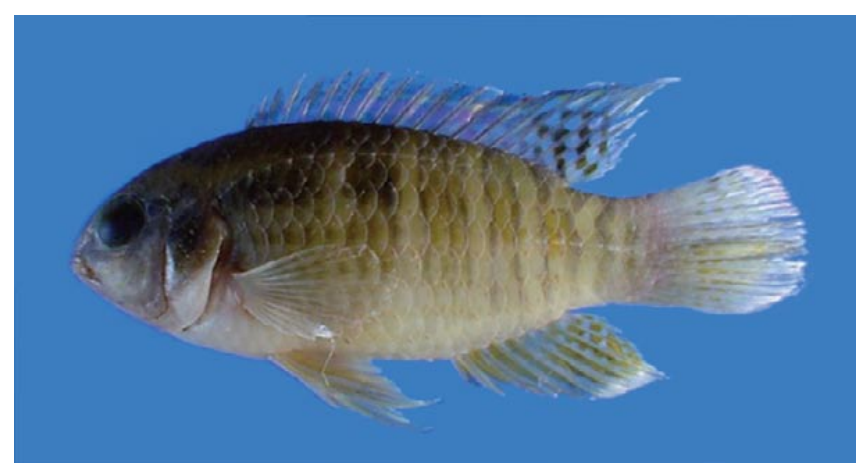

Figura 1. Laetacara aff. araguaiae, $42,2 \mathrm{~mm}$ de comprimento padrão (foto: F. Langeani).

Figure 1. Laetacara aff. araguaiae, $42.2 \mathrm{~mm}$ standard length (photo: F. Langeani). 


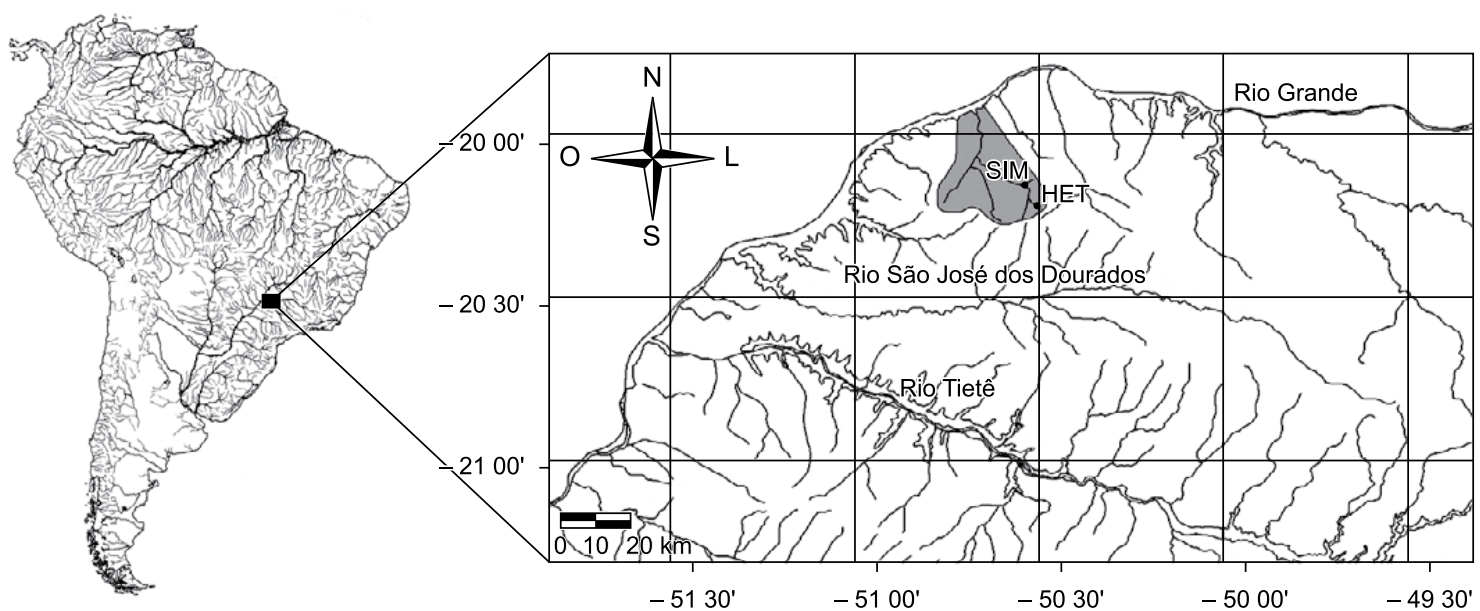

Figura 2. Localização geográfica dos riachos estudados (HET = Heterogêneo; SIM = Simplificado) na região Noroeste do Estado de São Paulo, sistema do alto Rio Paraná. A área em cinza representa a bacia do ribeirão do Arara.

Figure 2. Geographical location of the studied streams (HET = Heterogeneous; SIM = Simplified) in the Northwester region of the São Paulo State, upper Rio Paraná system. The grey area represents the ribeirão do Arara basin.

Tabela 1. Médias dos valores dos descritores limnológicos dos riachos heterogêneo (HET) e simplificado (SIM) durante os períodos chuvoso e seco.

Table 1. Average values of limnological descriptors in the heterogeneous (HET) and simplified (SIM) streams during wet and dry periods.

\begin{tabular}{lccccc}
\hline \multicolumn{1}{c}{ Descritores } & \multicolumn{2}{c}{ HET } & & \multicolumn{2}{c}{ SIM } \\
\cline { 2 - 3 } \cline { 5 - 6 } & Chuvoso & Seco & & Chuvoso & Seco \\
\hline Condutividade $\left(\mu \mathrm{S} . \mathrm{cm}^{-1}\right)$ & 0,09 & 0,08 & & 0,07 & 0,09 \\
Temperatura $\left({ }^{\circ} \mathrm{C}\right)$ & 31,1 & 23,2 & & 28,0 & 25,3 \\
Turbidez $(\mathrm{NTU})$ & 91 & 28 & & 18,3 & 11,3 \\
Volume $\left(\mathrm{m}^{3}\right)$ & 9,9 & 3,9 & & 8,5 & 5,7 \\
\hline
\end{tabular}

Para testar se as diferenças nos descritores limnológicos entre os riachos e nos parâmetros ecológicos entre as populações foram significativas, buscou-se primeiramente verificar a homogeneidade das variâncias por meio do teste de Levene para aplicação do teste $t$; quando esta premissa não foi atendida, o teste $t$ foi aplicado com a opção de variâncias separadas. Tais análises foram conduzidas com 5\% de nível de significância no programa STATISTICA 6.0.

\section{Resultados}

Foram constatadas poucas variações dos descritores limnológicos entre os dois riachos e entre períodos sazonais (Tabela 1). No riacho HET, durante o período chuvoso foram registrados o maior volume de hábitat e a maior temperatura da água (teste $t, \mathrm{p}_{\text {volume }}=0,025$, $\mathrm{p}_{\text {temperatura }}=0,037$ ), enquanto que os demais descritores não demonstraram variações estatisticamente significativas. Analisando o conjunto das seis repetições amostrais entre os riachos, somente a maior turbidez no riacho HET não ocorreu ao acaso (teste $t$, $\mathrm{p}=0,037)$.

Foram coletados 118 indivíduos no riacho HET, sendo 49 no período chuvoso (16,7-52,3 $\mathrm{mm} \mathrm{CP}$, mediana $37,8 \mathrm{~mm})$ e 69 no período seco $(10,6-58,6 \mathrm{~mm} \mathrm{CP}$, mediana $35 \mathrm{~mm})$. No riacho SIM foram capturados 146 espécimes, dos quais 82 no período chuvoso $(6,0-53,0 \mathrm{~mm} \mathrm{CP}$, mediana $26,4 \mathrm{~mm})$ e 64 no período seco (13,9-35,6 mm CP, mediana 25,2 $\mathrm{mm})$, demonstrando nitidamente que no riacho HET os indivíduos apresentaram maior porte do que no riacho SIM (Figura 3) e esta tendência não ocorre ao acaso
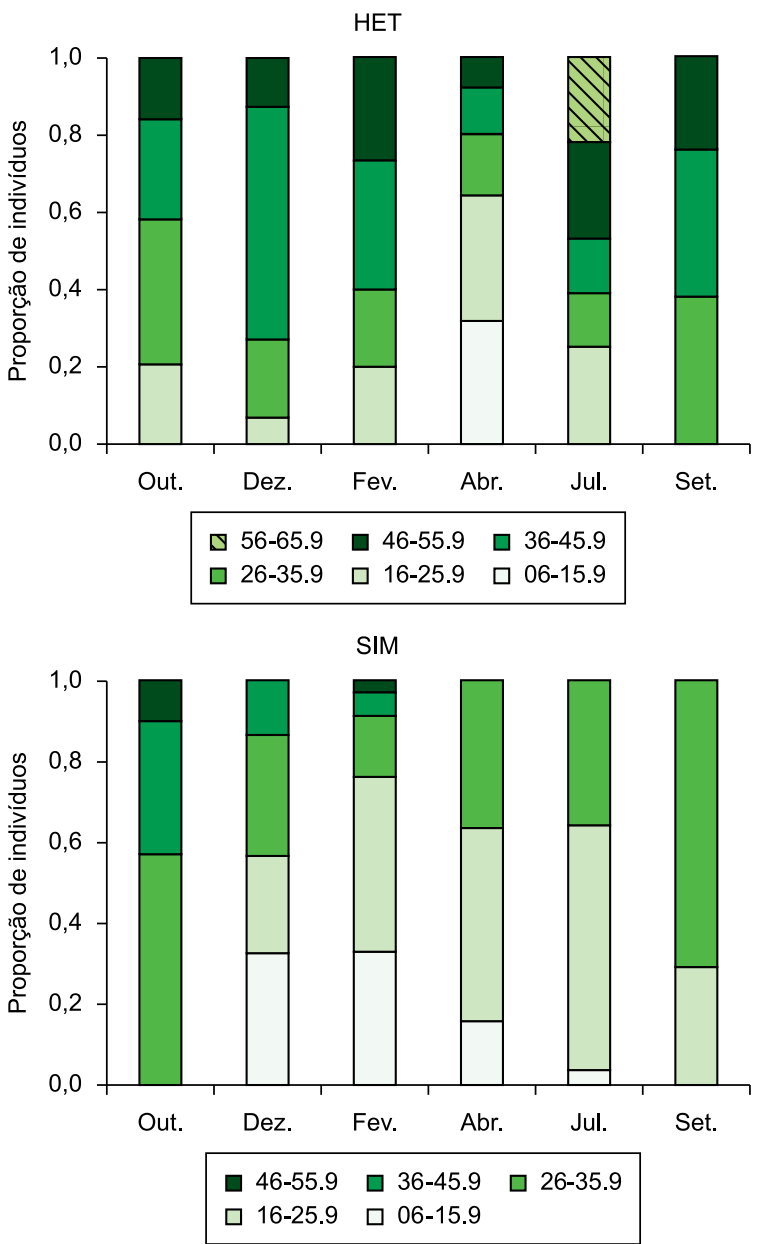

Figura 3. Proporção de indivíduos de Laetacara aff. araguaiae por classes de tamanho $(\mathrm{mm})$ em seis amostragens realizadas em dois riachos $($ HET $=$ Heterogêneo; SIM = Simplificado $)$ do Noroeste do Estado de São Paulo, Brasil.

Figure 3. Proportion of individuals of Laetacara aff. araguaiae by size classes $(\mathrm{mm})$ in six samplings in two streams (HET = Heterogeneous; SIM = Simplified) of the Northwestern of São Paulo State, Brazil. 
quando considerados os períodos sazonais (teste $t$, período chuvoso, $\mathrm{p}<0,0001$; período seco, $\mathrm{p}<0,0001$ ). A densidade de peixes nos dois ambientes foi mais elevada no período seco, com picos no mês de julho (Figura 4), porém essas diferenças ocorrem ao acaso tanto quando avaliados os períodos sazonais quanto os riachos (teste $t$, $\mathrm{p}>0,05)$.

No riacho HET foram examinados os conteúdos gástricos de 98 indivíduos (46 no período chuvoso e 52 no seco), com registro de oito tipos de itens alimentares, sendo seis deles de origem autóctone e dois, alóctone (Tabela 2). No período chuvoso, os itens mais importantes foram fragmentos de invertebrados aquáticos muito triturados (mas que foram passíveis de reconhecimento por apêndices locomotores, brânquias e peças do aparato mandibular), bem como algas e fragmentos de vegetais, caracterizando uma dieta onívora. No período seco, fragmentos de invertebrados aquáticos foram os itens mais importantes, seguidos por insetos aquáticos, indicando dieta invertívora (Figura 5). No riacho SIM foram examinados 108 indivíduos (55 no período chuvoso e 53 no seco), com registro de 10 tipos de itens alimentares (Tabela 2), sendo que os mais importantes, em ambos os períodos, foram fragmentos de invertebrados aquáticos e insetos aquáticos (Figura 5), indicando

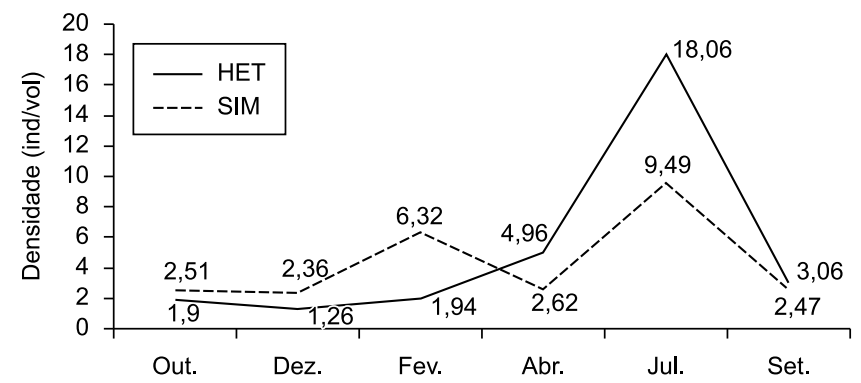

Figura 4. Densidade de indivíduos de Laetacara aff. araguaiae (por volume de hábitat) coletados em dois riachos $(\mathrm{HET}=$ Heterogêneo; $\mathrm{SIM}=$ Simplificado $)$ do Noroeste do Estado de São Paulo, Brasil.

Figure 4. Density of Laetacara aff. araguaiae (by habitat volume) individuals collected two streams $($ HET $=$ Heterogeneous; SIM $=$ Simplified $)$ of the Northwestern of São Paulo State, Brazil. dieta invertívora. Em ambos os riachos predominaram estômagos parcialmente cheios $(85,71 \%$ em HET; $86,84 \%$ em SIM).

A fecundidade foi significativamente maior no riacho HET (teste $t$, $\mathrm{p}<0,0001$ ), variando de 977 a 1.602 ovócitos, em contraste com as fêmeas do riacho SIM, que apresentaram de 338 a 814 ovócitos. Para a relação entre fecundidade e tamanho $(\mathrm{CP})$ obteve-se o valor médio de 21,4 para a amostra do riacho HET e de 30,3 para a amostra do riacho SIM, sendo que esta diferença não ocorre ao acaso (teste $t$, $p=0,007)$. Os ectoparasitos foram encontrados aderidos às nadadeiras (dorsal e anal) dos peixes em todas as amostragens no riacho SIM, com maior prevalência no mês de outubro (Tabela 3). Não foram registrados ectoparasitos em indivíduos do riacho HET.

\section{Discussão}

O maior volume de hábitat registrado nas três primeiras amostragens coincide com o período de maior pluviosidade na região (out.-fev.: 982,8 mm; abr.-set.: 71,4 mm, CIIAGRO 2008), período este em que se constatou a menor densidade de peixes em ambos os riachos, refletindo a bem conhecida relação entre espaço e abundância (Connor \& McCoy 1979, Whittaker 1998). Apesar desta variação, ao contrário do esperado, a densidade não variou entre os riachos HET e SIM. Segundo Teresa (2007), esta espécie ocorre preferencialmente em áreas marginais de riachos de leito arenoso, mostrando que não é uma espécie dependente dos bancos de vegetais submersos, como os presentes no riacho HET. Dessa forma, os elementos que conferem heterogeneidade ao riacho em questão não influenciariam diretamente a abundância destes peixes, mas sim, indiretamente, por meio da interferência nos recursos e condições, como, por exemplo, na turbidez, que foi significativamente maior no riacho HET. Ainda, os maiores valores de turbidez no riacho HET durante o período chuvoso coincidem com maior proliferação de algas filamentosas e macrófitas observadas in loco. Estes organismos promovem maior adensamento de estruturas submersas (raízes e filamentos), que são facilitadoras ao acúmulo de detritos (Rocha et al. 2009), explicando, portanto, a maior freqüência e dominância deste item nos estômagos provenientes do riacho HET no período chuvoso.

Os itens alimentares de origem autóctone foram de extrema importância para a dieta dos peixes, predominando nos dois riachos,

Tabela 2. Freqüência de ocorrência $(\% \mathrm{~F})$, dominância $(\% \mathrm{D})$ dos itens alimentares e grau de enchimento dos estômagos de indivíduos de Laetacara aff. araguaiae em dois riachos (HET = Heterogêneo; SIM = Simplificado) do Noroeste do Estado de São Paulo, Brasil, nos períodos chuvoso e seco.

Table 2. Frequency of occurrence $(\% \mathrm{~F})$, dominance $(\% \mathrm{D})$ of feeding items and degree of stomach fullness of Laetacara aff. araguaiae individuals in two streams (HET = heterogeneous; SIM = simplified) of Northwestern São Paulo State, Brazil, during wet and dry periods.

\begin{tabular}{|c|c|c|c|c|c|c|c|c|}
\hline \multirow[t]{3}{*}{ Itens } & \multicolumn{4}{|c|}{ HET } & \multicolumn{4}{|c|}{ SIM } \\
\hline & \multicolumn{2}{|c|}{ Chuvoso } & \multicolumn{2}{|c|}{ Seco } & \multicolumn{2}{|c|}{ Chuvoso } & \multicolumn{2}{|c|}{ Seco } \\
\hline & FO & $\mathbf{D}$ & FO & D & FO & $\mathbf{D}$ & FO & D \\
\hline Fragmentos de invertebrados & 76,1 & 32,6 & 80,8 & 50,0 & 89,1 & 67,3 & 90,6 & 60,4 \\
\hline Fragmentos de vegetais & 56,5 & 19,6 & 42,3 & 11,5 & 7,3 & 1,8 & 17,0 & 7,3 \\
\hline Insetos aquáticos & 41,3 & 6,5 & 51,9 & 21,1 & 65,4 & 14,5 & 62,3 & 23,6 \\
\hline Detritos & 10,9 & 8,7 & 7,1 & 7,7 & - & - & 3,8 & 3,8 \\
\hline Insetos terrestres & 2,2 & - & - & - & 3,6 & - & - & - \\
\hline Microcrustáceos & 43,5 & 8,7 & 38,5 & 7,7 & 36,4 & 12,7 & 26,4 & 3,8 \\
\hline Aracnídeos & - & - & 1,9 & - & 7,3 & - & 9,4 & - \\
\hline Hirudíneos & - & - & - & - & 1,8 & 1,8 & - & - \\
\hline Algas & 76,1 & 23,9 & 46,1 & 1,9 & 16,4 & 1,8 & 5,7 & - \\
\hline Escamas & - & - & - & - & - & - & 1,9 & - \\
\hline \multirow[t]{3}{*}{ Grau de enchimento estomacal } & \multicolumn{2}{|c|}{$1-0 \%$} & \multicolumn{2}{|c|}{$1-11,9 \%$} & \multicolumn{2}{|c|}{$1-5,2 \%$} & \multicolumn{2}{|c|}{$1-5,3 \%$} \\
\hline & \multicolumn{2}{|c|}{$2-91,3 \%$} & \multicolumn{2}{|c|}{$2-81,3 \%$} & \multicolumn{2}{|c|}{$2-86,2 \%$} & \multicolumn{2}{|c|}{$2-87,5 \%$} \\
\hline & \multicolumn{2}{|c|}{$3-8,7 \%$} & \multicolumn{2}{|c|}{$3-6,8 \%$} & \multicolumn{2}{|c|}{$3-8,6 \%$} & \multicolumn{2}{|c|}{$3-7,1 \%$} \\
\hline
\end{tabular}



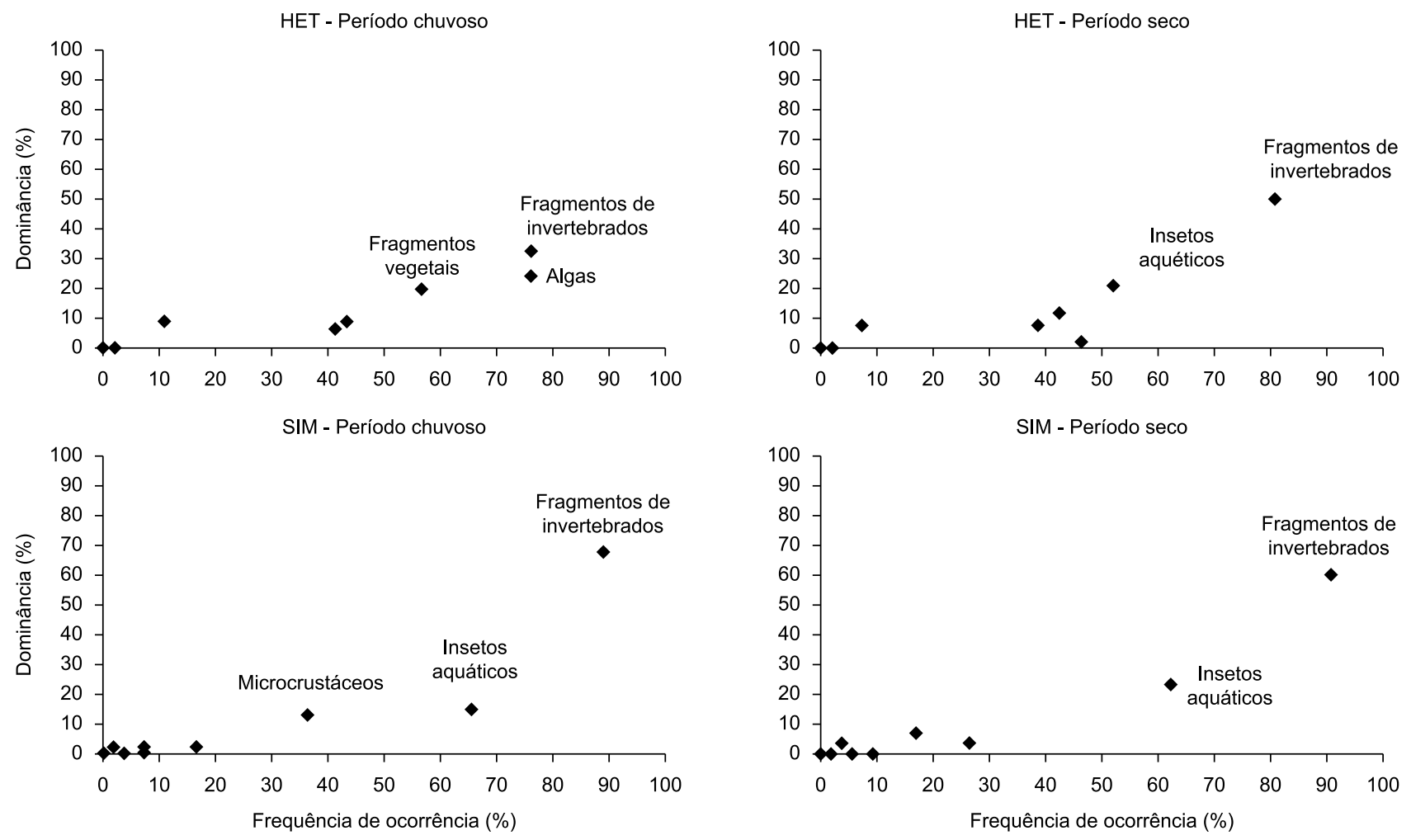

Figura 5. Itens alimentares preferenciais consumidos por indivíduos de Laetacara aff. araguaiae coletados em dois riachos (HET = Heterogêneo; SIM = Simplificado) do Noroeste do Estado de São Paulo, Brasil, nos períodos chuvoso e seco.

Figure 5. Main feeding items consumed by Laetacara aff. araguaiae individuals in two streams $(\mathrm{HET}=\mathrm{Heterogeneous;} \mathrm{SIM=Simplified)}$ of the Northwestern of São Paulo State, Brazil, during the wet and dry periods.

Tabela 3. Índices de parasitismo registrados nos peixes do riacho simplificado, Noroeste Do Estado de São Paulo, Brasil, nos períodos chuvoso (Out., Dez., Fev.) e seco (Abr., Jul., Set.)

Table 3. Parasitism indexes registered in the fishes of the simplified stretch, Northwestern São Paulo State, Brazil, during wet (Out., Dez., Fev.) and dry periods (Abr., Jul., Set.).

\begin{tabular}{llllllc}
\hline \multicolumn{1}{c}{ Índices } & Out. & Dez. & Fev. & Abr. & Jul. & Set. \\
\hline $\begin{array}{l}\mathrm{N}^{\mathbf{c}} \text { hospedeiros } \\
\text { examinados }\end{array}$ & 21 & 28 & 33 & 19 & 28 & 17 \\
$\begin{array}{l}\text { Abundância de } \\
\text { parasitos }\end{array}$ & 390 & 22 & 1 & 1 & 21 & 1 \\
$\begin{array}{l}\text { Abundância } \\
\text { média }\end{array}$ & 18,60 & 0,78 & 0,03 & 0,05 & 0,75 & 0,06 \\
$\begin{array}{l}\text { Intensidade } \\
\text { média }\end{array}$ & 22,9 & 5,5 & 1 & 1 & 2,6 & 1 \\
$\begin{array}{l}\text { Prevalência }(\%) \\
81\end{array}$ & 14 & 3 & 5 & 28 & 6 \\
\hline
\end{tabular}

independente do período sazonal. Fragmentos de invertebrados aquáticos foram os itens alimentares mais importantes em todas as amostras, sendo importante ressaltar que estes itens estavam sempre muito triturados. $\mathrm{O}$ estado de fragmentação do conteúdo estomacal pode estar relacionado ao fato dos peixes da Ordem Perciformes apresentarem numerosos e bem desenvolvidos dentes faringeanos em placas, que são responsáveis pela trituração do alimento antes da sua chegada ao estômago (Moyle \& Cech 2000).

Não foi possível atingir a identidade específica do parasito registrado, mas trata-se de uma espécie de digenético (Platyhelminthes) com ciclo heteroxênico, no qual os peixes participam como hospedeiros intermediários (Gilberto C. Pavanelli, inf. pessoal). Embora o registro de parasitos tenha ocorrido em todos os períodos amostrais no riacho SIM, a maior abundância e intensidade média nos meses de outubro e dezembro indicam que esta é a época mais favorável à sua reprodução. Como a espécie de parasito não foi identificada, não é possível inferir sobre a natureza da injúria ao hospedeiro, mas em razão do alto grau de infecção, provavelmente a alocação de recursos para manutenção seja maior na população dos peixes em SIM, em detrimento ao crescimento e reprodução. Estes fatores provavelmente podem explicar o menor porte e menor fecundidade dos peixes do riacho SIM quando comparado ao HET e, de fato, a menor fecundidade em SIM parece estar relacionada ao menor tamanho das fêmeas deste local. Wootton (1998) discute diversos aspectos qualitativos da interação entre o parasito cestóide Schistocephalus solidus e espécies de Gasterosteus, dentre eles o aumento do risco de predação, desfavorecimento na competição por recursos, inibição da maturação sexual e prejuízos na produção de ovos.

O uso diferencial do alimento poderia ser um fator que potencialmente explicaria as diferenças de tamanho corporal encontradas entre as duas populações; contudo, o grau de enchimento estomacal foi semelhante entre os peixes dos dois riachos, indicando quantias semelhantes na ingestão do alimento. Apesar das diferenças com relação à variedade de itens alimentares registrados nos conteúdos estomacais dos peixes de ambos os riachos, o mesmo item (fragmentos de invertebrados aquáticos) foi dominante, permitindo supor que a oferta alimentar seja equivalente nos dois ambientes, tanto do ponto de vista quantitativo quanto nutricional. Por outro 
lado, é provável que haja maior ocorrência de predação no riacho SIM, visto que este é mais raso, com água mais transparente e com menor quantidade de refúgios, dada a menor complexidade estrutural. Embora esta seja uma explicação razoável, são necessários estudos mais detalhados para investigá-la apropriadamente. Mais provável, contudo, é o prejuízo que parasitos alojados nas nadadeiras podem causar aos peixes, especialmente sobre deslocamentos natatórios, afetando assim, a performance durante a fuga contra predadores (Wootton 1998).

Em síntese, a alimentação e densidade foram similares nas duas populações de Laetacara aff. araguaiae, ao contrário da estrutura em tamanho e fecundidade. As diferenças nestes parâmetros demográficos não ocorrem ao acaso e são possivelmente reflexo das elevadas taxas de parasitismo no riacho mais simplificado que, em resumo, determinam menor alocação de recursos para crescimento e reprodução nesta população de peixes.

\section{Agradecimentos}

Fernando R. Carvalho e Oscar J. Canassa pelo apoio em campo, equipe do Laboratório de Ictiologia do IBILCE/UNESP pelo apoio em laboratório, Gilberto C. Pavanelli pelo auxílio com a identificação (via fotografia) do parasito, Francisco Langeani e Fernando R. Carvalho pela identificação dos indivíduos das duas populações, Fabrício B. Teresa e assessoria da Biota Neotropica pela leitura crítica do manuscrito e valiosas sugestões. Este trabalho foi financiado pela Fundação de Amparo à Pesquisa do Estado de São Paulo (FAPESP) dentro do Programa BIOTASP/FAPESP (www.biota. org.br) - O Instituto Virtual da Biodiversidade (processos 01/13340-7, 04/04820-3, 06/02276-0). PSSF é bolsista da FAPESP.LC é bolsista de Produtividade CNPq.

\section{Referências Bibliográficas}

ANGERMEIER, P.L. \& KARR, J.R. 1984. Fish communities along environmental gradients in a system of tropical streams. In Evolutionary ecology of Neotropical freshwater fishes (T.M. Zaret, ed.). Junk, The Hague, p.38-56.

ARID, F.M. 1973. Comportamento espacial da formação Bauru na região norte-ocidental do estado de São Paulo. Rev. Bras. Geoc. 3(1):23-24.

BENGTSSON, J. 1991. Interspecific competition in metapopulations. Biol. J. Linn. Soc. 42(1-2):219-237.

BENNEMANN, S.T., CASATTI, L. \& OLIVEIRA, D.C. 2006. Alimentação de peixes: proposta para análise de itens registrados em conteúdos gástricos. Biota Neotrop. 6(2): <http://www.biotaneotropica.org.br/v6n2/ pt/abstract?article+bn01206022006>. (último acesso em 11/05/2009).

BÜHRNHEIM, C.M. \& COX-FERNANDES, C. 2003. Structure of fish assemblages in Amazonian rain-forest streams: effects of habitats and locality. Copeia 2003(2):255-262.

BUSH, A.O., LAFFERTY, K.D., LOTZ, J.M. \& SHOSTAK, A.W. 1997. Parasitology meets ecology on its own terms: Margolis et al. revisited. J. Parasitol. 83(4):575-583.

CASATTI, L., LANGEANI, F. \& FERREIRA, C.P. 2006. Effects of physical habitat degradation on the stream fish assemblage structure in a pasture region. Environ. Manag. 38(6):974-982.

CONNOR, E.F. \& McCOY, E.D. 1979. The statistics and biology of the species-area relationship. Am. Nat. 113:791-833.

COSTELLO, M.J. 1990. Predator feeding strategy and prey importance: a new graphical analysis. J. Fish Biol. 36(2):261-263.

FLECKER, A.S. 1997. Habitat modification by tropical fishes: environmental heterogeneity and the variability of interaction strength. J. N. Am. Benthol. Soc. 16(1):286-295.
GELWICK, F.P. \& MATTHEWS, W.J. 1996. Trophic relations of stream fishes. In Methods in stream ecology (G. Lamberti \& R. Hauer, eds). Academic Press, New York, p.475-492.

GORMAN, O.T. \& KARR, J.R. 1978. Habitat structure and stream fish communities. Ecology 59(3):507-515.

GROSHOLZ, E.D. 1993. The influence of habitat heterogeneity on hostpathogen population dynamics. Oecologia 96(3):347-353.

HASTINGS, A. 1991. Structured models of metapopulation dynamics. Biol. J. Linn. Soc. 42(1-2):57-71.

HYNES, H.B.N. 1950. The food of fresh-water sticklebacks (Gasterosteus aculeatus and Pygosteus pungitius), with a review of methods used in studies of the food of fishes. J. Anim. Ecol. 19:36-57.

MATTHEWS, W.J. 1998. Patterns in freshwater fish ecology. Chapman \& Hall, New York.

McINTOSH, A.R., PECKARSKY, B.L. \& TAYLOR, B.W. 2004. Predatorinduced resource heterogeneity in a stream food web. Ecology 85(8):2279-2290.

MOYLE, P.B. \& CECH, J.J. 2000. Fishes: an introduction to ichthyology. Prentice Hall, Upper Saddle River.

NIMER, E. 1989. Climatologia do Brasil. IBGE, Rio de Janeiro.

PALMER, M.A. \& POFF, N.L. 1997. Heterogeneity in streams: the influence of environmental heterogeneity on patterns and processes in streams. J. N. Am. Benthol. Soc. 16(1):169-173.

ROCHA, F.C., CASATTI, L. \& PEREIRA, D.C. 2009. Structure and feeding of a stream fish assemblage in Southeastern Brazil: evidence of low seasonal influences. Acta Limnol. Bras. 21(1):123-134.

São Paulo (Estado). Centro Integrado de Informações Agrometeorológicas CIIAGRO. <http://www.ciiagro.sp.gov.br/ciiagroonline/> (último acesso em 30/04/2008).

SCHNEIDER, K.N. \& WINEMILLER, K.O. 2008. Structural complexity of woody debris patches influences fish and macroinvertebrate species richness in a temperate floodplain-river system. Hydrobiol. 610(1):235-244

SIMPSON, K., McCAULEY, E. \& NELSON, W.A. 2008. Spatial heterogeneity and rates of spread in experimental streams. Oikos 117(10):1491-1499.

SNYDER, C.D., YOUNG, J.A. \& STOUT III, B.M. 2006. Aquatic habitats of Canaan valley, West Virginia: Diversity and environmental threats. Northeast. Nat. 13(3):333-352.

TAYLOR, A.D. 1990. Metapopulations, dispersal, and predator-prey dynamics: an overview. Ecology 71:429-433.

TERESA, F.B. 2007. Comportamento reprodutivo e cooperação no ciclídeo neotropical Laetacara sp. Dissertação de Mestrado, Universidade Estadual Paulista, São José do Rio Preto.

THEEL, H.J., DIBBLE, E.D. \& MADSEN, J.D. 2008. Differential influence of a monotypic and diverse native aquatic plant bed on a macroinvertebrate assemblage; an experimental implication of exotic plant induced habitat. Hydrobiol. 600(1):77-87.

TORGERSEN, C.E. \& CLOSE, D.A. 2004. Influence of habitat heterogeneity on the distribution of larval Pacific lamprey (Lampetra tridentata) at two spatial scales. Fresh. Biol. 49(5):614-630.

WHITTAKER, R.J. 1998. Island biogeography: ecology, evolution, and conservation. Oxford University Press, Oxford.

WOOTTON, R.J. 1998. Ecology of teleost fishes. 2 ed. Kluwer Academic Publishers, London. 